\title{
Transposição da valva pulmonar para substituição da valva aórtica
}

\author{
Henrique B. FURTADO*, Carlos A. DURAN*, Gonzalo H. MEJIAS*, Nilton BITTENCOURT*, \\ Reginaldo A. BARROS*, Antônio Carlos B. CAMARGO*, Antônio E. GERMANO*
}

RBCCV 44205-120

\begin{abstract}
FURTADO, H. B.; DURAN, C. A.; MEJIAS, G. H.; BITTENCOURT, N.; BARROS, R. A.; CAMARGO, A. C. B.; GERMANO, A. E. - Transposiçảo da valva pulmonar para substituiçāo da valva aórtica. Rev. Bras. Cir. Cardiovasc, 5(3): 160-167, 1990.

RESUMO - Embora tenha havido uma importante melhora na qualidade das próteses cardíacas aórticas, existem, ainda, problemas consideráveis a longo prazo, como o uso de aticoagulantes para as próteses mecânicas e a durabilidade dos tecidos biológicos fixados e mortos. No presente trabalho, relatamos as técnicas utilizadas para se transplantar um tubo valvulado obtido pela dissecçāo da valva e tronco pulmonar do próprio paciente e seu implante no local da valva aórtica suturada sob os óstios das artérias coronárias direita e esquerda. Após isto, reconstruímos a via de saída de ventrículo direito (VSVD), valva e tronco pulmonar com tubo valvulado de pericárdio bovino. O paciente teve ótima evoluçāo pós-operatória, com a neovalva aórtica perfeitamente competente e bom fluxo pulmonar. O pericárdio bovino utilizado em posição pulmonar tem uma tendência desprezível de complicaçōes a longo prazo bem menor que em posiçāo aórtica e a valva pulmonar homóloga viva deverá ser definitiva.
\end{abstract}

DESCRITORES: próteses valvulares cardíacas, biológicas; auto-enxerto pulmonar, cirurgia.

\section{INTRODUÇĀO}

A busca do substituto valvar ideal vem estimulando, nos últimos anos, o aparecimento de próteses cada vez mais perfeitas no que diz respeito ao seu desempenho hemodinâmico, histocompatibilidade e durabilidade 4.11.

Se, de um lado, as próteses metálicas nos oferecem uma grande durabilidade, de outro ainda não se conseguiu um material biocompatível a ponto de se evitar que fenômenos trombogênicos ocorram em grande quantidade, sendo necessário o uso definitivo de anticoagulantes orais, com seus conhecidos riscos e inconvenientes.

Desde as primeiras biopróteses, produzidas a partir da fascia lata, passando pela criativa prótese de dura-máter homóloga idealizada por PUIG et alii ${ }^{11}{ }^{12}$, as mais atuais sāo as biopróteses hete- rólogas de porco e de pericárdio bovino.

O desenvolvimento de suportes flexíveis de delrin, produzidos em nosso meio ${ }^{1.6}$, associado ao tratamento do tecido biológico pelo glutaraldeído (GDA), segundo as técnicas preconizadas por IONESCU et alii ${ }^{7}$, aumentou substancialmente a durabilidade dos biopróteses.

Entretanto, a degeneraçāo dos tecidos heterólogos, embora tardia, continua a ocorrer em número crescente após alguns anos.

Dentre as biopróteses, as heterólogas de porco tornaram-se muito populares. Elas apresentam bom desempenho hemodinâmico, sendo moderadamente estenóticas somente em diâmetros menores que $19 \mathrm{~mm}$ que oferecem uma área orificial menor que $1 \mathrm{~cm}^{2}$, com gradiente transvalvar que varia de 20 a $50 \mathrm{mmHg}$ ?. A maior limitação dessas próteses

Trabalho realizado no Instituto de Hemodinâmica e Cirurgia Cardiovascular e no Hospital de Base da Associação Hospitalar de Bauru. Bauru, SP, Brasil.

Apresentado ao $17^{\circ}$ Congresso Nacional de Cirurgia Cardiaca. Belo Horizonte, MG, 6 e 7 de abril, 1990.

* Do instituto de Hemodinâmica e Cirurgia Cardiovascular.

Endereço para separatas: Henrique B. Furtado. Rua Agenor Meira, 14, Centro. 17015 Bauru, SP. Brasil. 
FURTADO, H. B.; DURAN, C. A.; MEJIAS, G. H.; BITTENCOURT, N.; BARROS, R. A.; CAMARGO, A. C. B.; GERMANO, A. E. - Transposição da valva pulmonar para substituição da valva aórtica. Rev. Bras. Cir. Cardiovasc, 5(3): 160$167,1990$.

corresponde à degeneração do tecido biológico, levando a estenose ou insuficiência. Apesar dos procedimentos de controle de qualidade na produção, incluindo o tratamento dos tecidos sob baixa pressăo, uso de tampão biológico e inúmeros outros cuidados, vários trabalhos demonstram que a durabilidade dessas próteses, após 10-12 anos, ainda nāo é ideal.

CRAVER et alii ${ }^{4}$, em trabalho realizado na Emory University, demonstram que, em mais de 1000 pacientes observados, havia um aumento significativo de disfunção após seis anos, principalmente nos pacientes com menos de 35 anos de idade.

JAMIESON et alii ${ }^{8}$ demonstraram, em 1988, num grupo de 1183 pacientes com biopróteses de Carpentier Edwards, que o tempo médio de falha era de 74 meses. A análise atuarial de pacientes livres de degeneração da prótese em 10 anos foi de $(77,4 \% \pm 3 \%)$ para os pacientes com 35 a 60 anos e de $(26,8 \% \pm 17,2 \%)$ para menores que 35 anos. A curva atuarial demonstra uma queda brusca entre oito a dez anos.

O trabalho de FOSTER et alii ${ }^{5}$, com 10-15 anos de follow-up, demonstra que o tempo médio de falha está em $8,6 \pm 2,5$ anos.

Em posiçāo aórtica, apesar dos bons resultados iniciais, a falha primária do tecido ocorre, sendo que, aos seis anos, $97 \% \pm 2 \%$ estão livres de complicaçōes; este número cai para $68 \% \pm 8$ em pacientes com idade acima de 40 anos com 12,5 anos de evolução.

Outras biopróteses largamente difundidas correspondem as biopróteses de pericárdio bovino fixado em glutaraldeído (GDA), idealizadas por IONESCU et alii ${ }^{7}$. Os resultados iniciais são comparáveis à válvula de porco; porém, como o tecido biológico é montado fora do anel, elas apresentam uma discreta vantagem hemodinâmica sobre as válvulas porcinas nos diâmetros menores que $19 \mathrm{~mm}$.

SPAMPINATO et alii ${ }^{19}$ estudaram um grupo de pacientes com sete tipos diferentes de válvulas porcinas, na maioria Carpentier-Edwards, Hancock e Liotta, encontrando resultados semelhantes (Gráfico 1).

Pode-se inferir, portanto, que hoje possuímos próteses heterólogas de boa qualidade, do ponto de vista hemodinâmico, de histocompatibilidade e com uma sensível melhora de durabilidade até o limite de 10 a 12 anos. Entretando, nos pacientes menores que 35 anos, ou em paciente com expectativa de vida maior de 12 anos, devemos persistir na busca de um substituto valvar mais duradouro.

Sem dúvida, nenhuma prótese consegue supe-

\section{GRÁFICO 1}
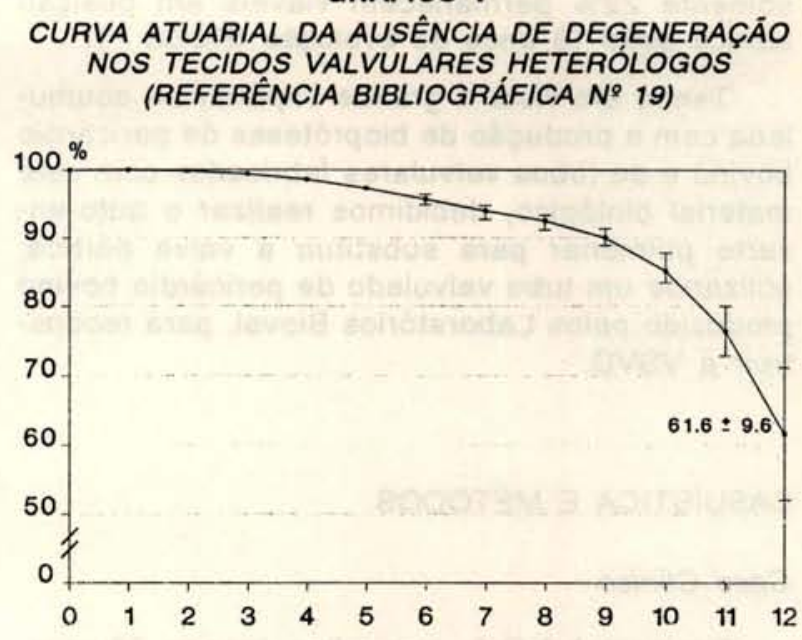

rar, sob qualquer ponto de vista, a performance da própria valva humana. Os problemas sempre se relacionaram com a técnica cirúrgica para seu implante e com os métodos de preservação e esterilizaçāo a serem utilizados.

Os primeiros implantes de homoenxerto com sucesso foram realizados por ROSS et alii ${ }^{15,18} \mathrm{e}$ BARRATT-BOYES ${ }^{3}$, cujos trabalhos determinam, até hoje, as técnicas de implante a serem utilizadas.

Foram implantados homoenxertos frescos (4.OC) e esterilizados em antibióticos e, muito embora os resultados iniciais tenham sido melhores que para as válvulas mecânicas ou biológicas, após 14 anos as curvas atuariais demonstram uma incidência de disfunção ao redor de $62 \%$, numa curva semelhante à que ocorre com a bioprótese porcina e de pericárdio bovino.

A aplicação dos homoenxertos, entretanto, também apresenta problemas relacionados com obtenção de doadores, estocagem, esterilizaçāo, discrepâncias de tamanho e de técnica cirúrgica.

Em 1967, ROSS ${ }^{17}$ descreveu a possibilidade de se utilizar a valva pulmonar homóloga para substituição da válva aórtica.

Sabendo-se que o resultado tardio, com o uso de homoenxerto vivo criopreservado, é bem superior ao das próteses biológicas citadas, pode-se supor que autilização do tecido do próprio coraçāo do paciente dará ótimos resultados. Entretanto, segundos STELZER \& ELKING ${ }^{20}$, não haveria a possibilidade de se transportar a valva pulmonar sem a existência de homoenxerto para reconstruçāo da VSVD, onde, pela baixa pressāo, os problemas com o enxerto seriam mínimos.

Sabe-se que $55 \%$ dos homoenxertos não se degeneram após 17 anos em posiçāo pulmonar e 
FURTADO, H. B.; DURAN, C. A.; MEJIAS, G. H.; BITTENCOURT, N.; BARROS, R. A.; CAMARGO, A. C. B.; GERMANO, A. E. - Transposiçāo da valva pulmonar para substituiçāo da valva aórtica. Rev. Bras. Cir. Cardiovasc, 5(3): 160$167,1990$.

somente $23 \%$ permanecem viáveis em posição aórtica após 15 anos de evolução (ROSS ${ }^{14}$ ).

Tendo em vista a grande experiência acumulada com a produção de biopróteses de pericárdio bovino e de tubos valvulares fabricados com este material biológico, decidimos realizar o auto-enxerto pulmonar para substituir a valva aórtica, utilizando um tubo valvulado de pericárdio bovino produzido pelos Laboratórios Bioval, para reconstruir a VSVD.

\section{CASUÍSTICA E MÉTODOS}

\section{Caso Clínico}

Paciente A.A.S.A., masculino, branco, 38 anos, $54 \mathrm{~kg}$, com história de dispnéia que há dois anos progrediu dos grandes para os mínimos esforços (classe III/IV da NYHA). Há dois meses apresentando dor retro-esternal também relacionada aos esforços físicos, além de tonturas com escurecimento da visão. Nega antecedentes de poliartrite migratória ou outros sintomas de febre reumática. Fumante e etilista moderado.

Exame Físico: R.E.G. afebril, PA 120/70, FC 88 bpm, sem estase jugular. Frêmito sistólico no foco Ao $++/+++$. Ictus palpável (três polpas digitais) ao nível da linha hemiclavicular $E$.

À ausculta, sopro holossistólico +++/++++ em FAo e FAo $A C$ com irradiação para fúrcula e carótidas. Abdômen flácido, figado e baço não palpáveis. RX tórax: $A C>++/+++$ (Figura 1). Ao alongada. ECG: RS FC 75 bpm. BCRD e HBAE (Figura 2).

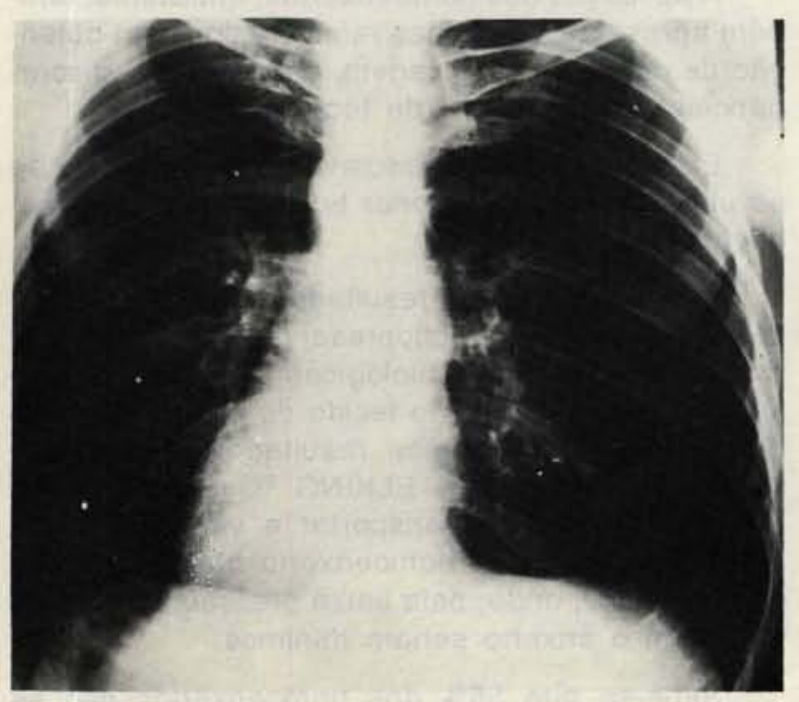

Fig. 1
Exames laboratoriais pré-operatórios: n.d.n. Reação de Machado Guerreiro e imunofluorescência para Chagas: negativos. Cateterismo cardíaco:

pressōes: VE - 200/14

$$
\text { G S }-90 \mathrm{mmHg}
$$

$$
\text { Ao }-110 / 70
$$

Conclusōes:1) VE hipertrófico; 2) valva mitral normal; 3) aparelho valvar aórtico calcificado com obstrução importante ao fluxo sangüíneo e discreto refluxo para o VE (DLAo tipo E Ao calcificada); 2) artérias coronárias normais.

Indicação: cirurgia para troca da valva aórtica.

\section{Cirurgia}

Paciente foi submetido a toracotomia mediana transesternal com pericardiotomia em $Y$ invertido. $O$ coração mostrava aorta aumentada de tamanho (distreta dilatação pós-estenótica) com frêmito sistólico +++/++++ na projeção da valva aórtica. Valva pulmonar e tronco pulmonar com cerca de $20 \mathrm{~mm}$. Em virtude da compatibilidade de diâmetro da valva pulmonar com o anel da valva aórtica, decidimos realizar a transposição. Instalamos CEC sob heparinização sistêmica e hipotermia moderada a $28^{\circ} \mathrm{C}$. Hipotermia tópica com gelo amorfo produzido em cardiofreezer e proteçāo frênica por tela termostática.

Clampeada a aorta, realizamos a aortotomia oblíqua em direção à parte média da válvula nāo coronariana. Cardioplegia clássica nos óstios das artérias coronárias D e E. A valva aórtica apresentava espessamento importante, com fusão comissural e intensa calcificação das três válvulas ( $\mathrm{Fi}$ gura 3). Retirada a valva aórtica e descalcificado - anel valvar. Havia cálcio em chama de vela sobre a cúspide anterior da valva mitral, que foi facilmente descalcificada. Dissecamos o tronco pulmonar (TP) até a bifurcação distalmente. Aberto o TP, examinamos a sua valva, que se apresentava com características normais. Feita incisăo cerca de $5 \mathrm{~mm}$ do anel válvar na VSVD. Retiramos a valva pulmonar conservando um bulbo de músculo do VD de cerca de $5 \mathrm{~mm}$ (Figura 4). Conferida a medida do anel que se fez compatível como o anel da valva aórtica. Passados 34 pontos simples com fio de polipropileno 4-0 no anel aórtico e no anel muscular do auto-enxerto, conservando os óstios coronários nos fundos de seio de Valsalva. Anudados os fios. Passados três pontos nas comissuras e na aorta, cerca de $10 \mathrm{~mm}$ acima do ponto comissural natural. Ressecção do excesso de TP, acompanhando o seio de Valsalva. Sutura em chuleio simples com fio de polipropileno 4-0, 
FURTADO, H. B.; DURAN, C. A.; MEJIAS, G. H.; BITTENCOURT, N.; BARROS, R. A.; CAMARGO, A. C. B.; GERMANO, A. E. - Transposiçāo da valva pulmonar para substituição da valva aórtica. Rev. Bras. Cir. Cardiovasc, 5(3): 160$167,1990$.
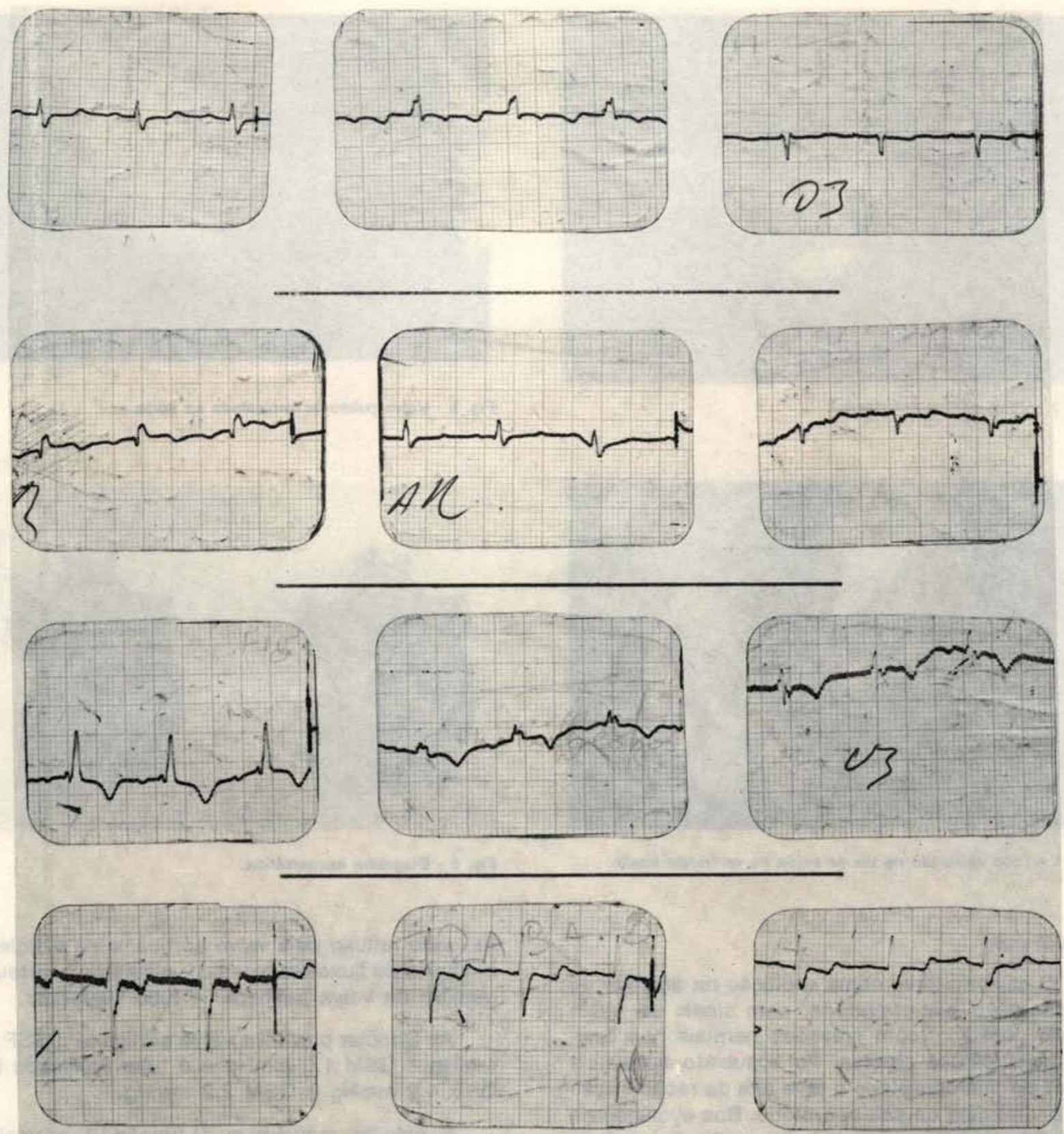

Fig. 2 - Eletrocardiograma.

deixando livre os óstios das artérias coronárias $D$ e E (Figura 5). Aortorrafia incluindo o auto-enxerto. Amarrados os pontos comissurais por fora da aorta. Retirando o ar das cavidades E e desclampeada a aorta. Realizada hemostasia da regiāo de dissecção na parede do VD (parede posterior). Clampeada aorta. Cardioplegia e hipotermia tópica. Sutura proximal de tubo valvulado de pericárdio bovino n. 21, modelo Bioval, com polipropileno 40 em chuleio contínuo. Anastomose distal do tubo com o tronco pulmonar com fio de sutura seme-
Ihante. Após retirada do ar das cavidades $D$ e $E$ e desclampeada a aorta, o coração recuperou-se em ritmo juncional, que evoluiu para ritmo sinusal. O tempo de CEC foi de $210^{\prime}$ e $183^{\prime}$ de clampeamento aórtico. $O$ paciente saiu de perfusão com PAM $80 \mathrm{mmHg}$, PVC $15 \mathrm{~cm} \mathrm{H20}$, boa diurese, FC $100 \mathrm{bpm}$. Retiradas as cânulas e amarradas as suturas em bolsa. Cuidadosa revisão da hemostasia. Fechamento de rotina do tórax, com drenagem tubular do mediastino. A figura 6 mostra, em esquema, o resultado final. 
FURTADO, H. B.; DURAN, C. A.; MEJIAS, G. H.; BITTENCOURT, N.; BARROS, R. A.; CAMARGO, A. C. B.; GERMANO, A. E. - Transposiçāo da valva pulmonar para substituição da valva aórtica. Rev. Bras. Cir. Cardiovasc, 5(3): 160$167,1990$.

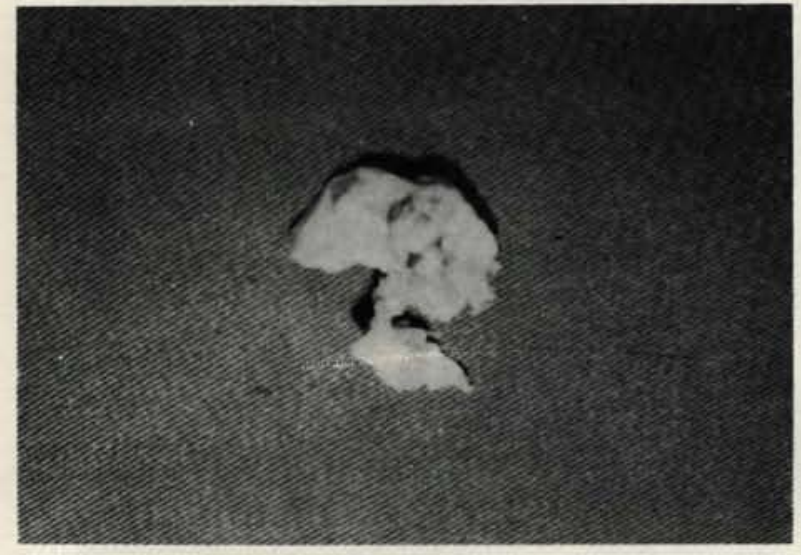

Fig. 3 - Valva aórtica calcificada.

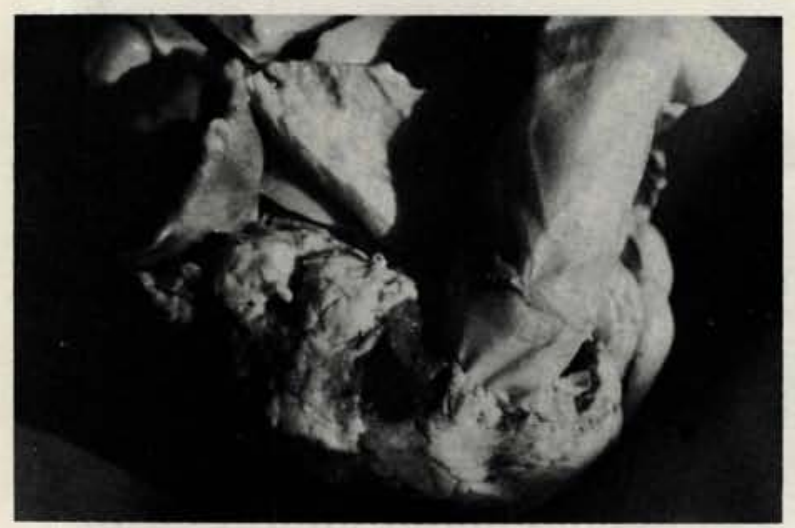

Fig. 4 - Tubo valvulado na via de saída de ventrículo direito.

\section{Resultados}

O paciente teve ótima evolução na unidade de recuperação pós-operatória, sem sinais de baixo débito cardíaco, com pressōes normais nas cavidades cardíacas direitas. Foi extubado após seis horas de pós-operatório e teve alta da recuperaçāo após 52 horas de pós-operatório. Boa evoluçāo na enfermaria tendo alta hospitalar no 90 dia de P.O.

Ambulatoriamente: após 60 dias da operação, encontra-se assintomático.

Ao exame: apresenta-se corado, eupnéico, sem sinais de insuficiência cardíaca, PA 120/80, FC 80. Coração com BRNF sem sopros. Abdômen flácido. $\mathrm{RHA}+$. Fígado e baço nảo palpáveis. $\mathrm{RX}$ tórax: normal. Fios metálicos no esterno. ECG: ritmo sinusal; FC 80; BCRD; HBAE. O estudo ecodopplercardiográfico mostra cavidades com volumes normais; VE hipertrófico com boa contratilidade; năo há gradientes sistólicos através do tubo valvulado e valva aórtica, sem refluxos. A Figura 7 (A e B) mostra enchimento do VE, em diástole plena, nāo

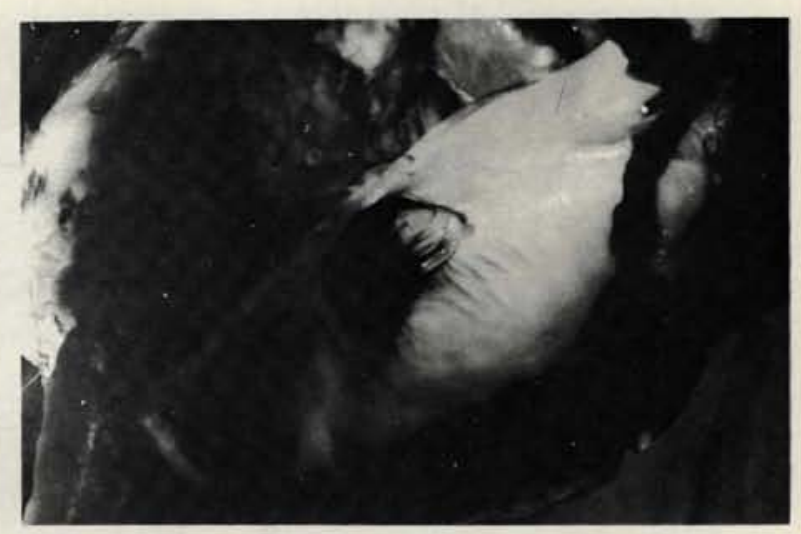

Fig. 5 - Valva pulmonar implantada na aorta.

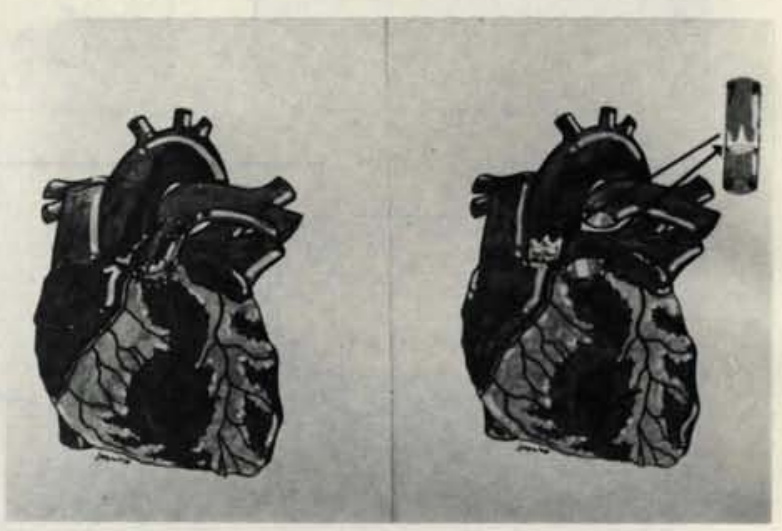

Fig. 6 - Diagrama esquemático.

havendo refluxo pela valva aórtica, e na sístole há ausência de fluxo em mosáico; o mesmo ocorreu no registro da valva pulmonar e tubo valvulado.

Ao Doppler pulsátil a válva aórtiça tem GSP 2,2 $\mathrm{mmHg}$ e GSM $1,0 \mathrm{mmHg}$ e o tubo valvulado tem GSP $4,2 \mathrm{mmHg}$ e GSM $2,2 \mathrm{mmHg}$.

Estudo hemodinâmico: 1) função VE normal; 2) ausência de insuficiência aórtica; 3) artérias coronárias E e D normoposicionadas; 4) VSVD e tubo valvular pulmonar com boa morfologia.

\section{COMENTÁRIOS}

A cirurgia de utilização do auto-enxerto pulmonar para substituir a valva aórtica, embora proposta há mais de 20 anos por ROSS ${ }^{17}$, no Reino Unido, não foi amplamente realizada, por alguns problemas, passiveis de discussāo: 1) ausência ou dificuldade de obtenção dọs homoenxertos para reconstrução da VSVD; 2) o receio de transformar uma 
FURTADO, H. B.; DURAN, C. A.; MEJIAS, G. H.; BITTENCOURT, N.; BARROS, R. A.; CAMARgo, A. C. B.; GERMANO, A. E. - Transposiçāo da valva pulmonar para substituição da valva aórtica. Rev. Bras. Cir. Cardiovasc, 5(3): 160$167,1990$.
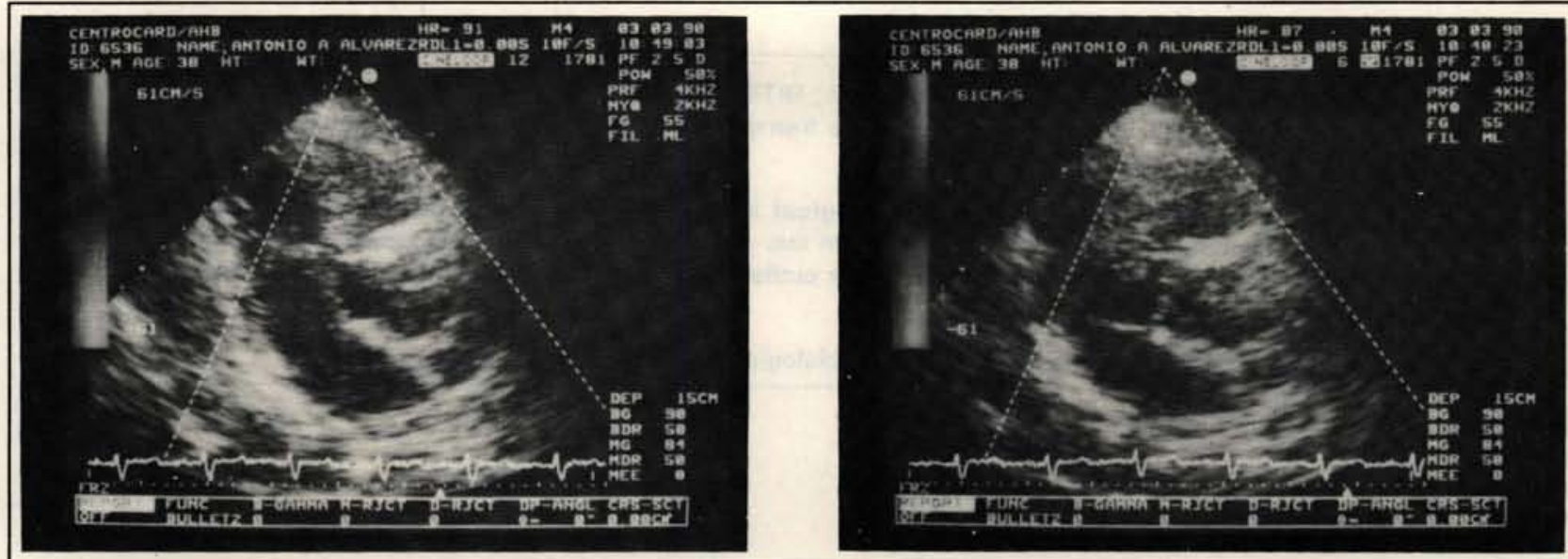

Fig. 7 A e B - Ecodopplercardiograma pós-operatório.

cirurgia de prótese aórtica, relativamente simples, em uma complexa cirurgia de dupla troca, com reconstruçăo da VSVD; 3 ) em 1971, McGOON ${ }^{2}$ afirmou achar que esta cirurgia realmente era interessante, porém temia que poucos cirurgiōes pudessem realizá-la, pela complexidade de se dissecar o tronco pulmonar na parede posterior, muito próximo ao tronco da $C E$ e os primeiros ramos septais; 4) a dificuldade no próprio implante, que pode deixar esta valva mal posicionada.

No presente trabalho, estamos tentando demonstrar que a maioria destes problemas já podem ser superados: 1) a experiência acumulada, no nosso meio, com os tubos valvulados de pericárdio bovino nos permite superar a falta dos homoenxertos, que também já estāo disponíveis. Acredito ter sido esta a primeira vez em que se realizou a cirurgia de auto-enxerto pulmonar com reconstruçāo da VSVD com tubo valvulado de pericárdio bovino; 2) a "complexidade cirúrgica" também já se encontra superada, graças à experiência com outras operaçōes semelhantes, como as derivaçōes átrio-pulmonares com utilização do tronco pulmonar e também em virtude da evolução dos métodos de proteçāo miocárdica.

Com relação às vantagens desta cirurgia, elas são incontestes:

A função da valva pulmonar é perfeita em posição aórtica, conforme experiência nesta operação e, mesmo, nos pacientes submetidos a cirurgia de Jatene para TGVB, (ARENSMAN et alii ${ }^{2}$ ).

A sua utilizaçāo em crianças oferece a única chance de uma prótese que poderá crescer com o crescimento da criança. Devemos apenas ter o cuidado de oferecer um tubo valvulado adequado na VSVD.
Se houver problemas futuros com o tubo valvulado, o que raramente ocorre, reoperar-se-á a VSVD, o que, sem dúvida, é muito mais simples.

O auto-enxerto é totalmente isento de trombogenicidade. Nāo houve nenhum caso de degeneraçāo do seu tecido, em 20 anos de evoluçāo, e é, sem dúvida, o substituto valvular ideal para a valva aórtica onde as outras próteses ainda sāo problemáticas.

Além de apresentar a forma semilunar natural, com ausência de fluxo turbilhonar, ausência de aneis e outros materiais sintéticos, nāo apresentam qualquer risco de resposta imunológica. Esta prótese é implantada com todas as suas células intáctas, inclusive o endotélio e os fibroblastos, sem qualquer tratamento conservante com antibióticos ou outros agentes químicos ${ }^{13}$.

Sem dúvida, para pacientes com lesāo valvar aórtica isolada, com expectativa de vida de 20 anos ou mais, deve-se considerar a possibilidade de realização desta cirurgia ${ }^{16}$.

\section{CONCLUSĀO}

A utilizaçāo do auto-enxerto pulmonar para pacientes que necessitarāo de sua valva aórtica por 20 anos ou mais no pós-operatório deve ser sempre considerada.

Demonstra-se que esta cirurgia pode ser realizada perfeitamente, utilizando-se tubo valvulado de pericárdio bovino.

Superados os problemas técnicos, o auto-enxerto pulmonar se constitui no substituto valvular ideal para a posição aórtica, nos casos em que ele estiver indicado. 
FURTADO, H. B.; DURAN, C. A.; MEJIAS, G. H.; BITTENCOURT, N.; BARROS, R. A.; CAMARGO, A. C. B.; GERMANO, A. E. - Transposiçāo da valva pulmonar para substituiçāo da valva aórtica. Rev. Bras. Cir. Cardiovasc, 5(3): 160$167,1990$.

\section{RBCCV 44205-120}

FURTADO, H. B.; DURAN, C. A.; MEJIAS, G. H.; BITTENCOURT, N.; BARROS, R. A.; CAMARGO, A. C. B.; GERMANO, A. E. - Pulmonary valve transposition for aortic valve replacement. Rev. Bras. Cir. Cardiovasc., 5(3): 160-167,1990.

ABSTRACT: Although there have been a great improvement in valvular prosthesis we still have problems concerning durability and thrombosis. In this paper we describe the transposition of pulmonary valve to aortic position with reconstruction of the outflow tract with a pericardial valvular tube, with very good results, in one patient.

DESCRIPTORS: heart valves prostheses, biologic; pericardial valvular tube, surgery.

\section{REFERÊNCIAS BIBLIOGRÁFICAS}

1 ABDULMASSIH NETO, C.; PAULISTA, P. P.; SOUZA, L. C. B.; MAGALHÄES, H. M.; MENEGHELO, Z. M.; LOURENÇO, A.; RICHTER, I.; ALIAGA, F. P.; JATENE, A. D. - Experiência clínica com bioprótese de pericárdio bovino. Arq. Bras. Cardiol., 41: $265.270,1983$.

2 ARENSMAN, F. W.; SIEVERS, H.; LANG. P. - Assessment of coronary and aortic anastomoses after anatomic corretion of transposition of the great arteries. $\mathrm{J}$. Thorac. Cardiovasc. Surg., 90: 597-604, 1985.

3 BARRATT-BOYES, B. G. - A method for preparing and inserting a homograft aortic valve. Br. J. Surg., 52 : 847-856, 1965.

4 CRAVER, J. M.; JONES, E. L.; McKEOWN, P. - Porcine cardiac xenograft valves: analysis of surviral, valve failure, and explantation. Ann. Thorac. Surg., 34:16$31,1982$.

FOSTER, A. H.; GREENBERG, G. J.; UNDERHILL, D. J. - Intrinsic failure of Hancock mitral bioprosthesis: 15 years experience. Ann. Thorac Surg., 44: 560$577,1987$.

6 FURTADO, H. B.; ABDULMASSIH NETO, C.; ARNONI, A. S.; CHACCUR, P.; OLIVEIRA, L. A. S.; PAULISTA, P. P.; SOUZA, L. C. B.; JATENE, A. D. - Confecçăo de válvula de pericárdio bovino sem sutura aparente com anel flexivel de delrin. Arq. Bras. Cardiol., 34 (Supl. 1): 25, 1980. (Resumo).

7 IONESCU. M.; SMITH, D. R.; CHIDONBORAN, M.; TANDON, A. P. - Clinical durability of the pericardial xenograft valve: ten years experience with mitral replacement. Ann. Thorac. Surg., 34: 265-277, 1982.

JAMIESON, W. R. E.; ROSADO, L. J.; MUNRO, A. I. - Carpentier - Edwards standard porcine bioprosthesis: primary tissue failure (structural valve deterioration) by age groups. Ann. Thorac. Surg., 46: 155$162,1988$.
9 McGOON, D. C. - Choice of grafts or prostheses for valvular replacement. Br. Heart J., 33 (Supl.): 35 38, 1971.

10 MAGILLIGAN Jr., D. J. - Porcine bioprosthesis, Cardiac Surg., 2: 269-284, 1987.

11 PUIG, L. B.; VERGINELLI, G.; KAUABE, L.; OLIVEIRA H. A.; POMERANTZEFF, P.; TAKEDA, R.; SOSA, E. SOUZA, M. C.; PILEGGI, F.; ZERBINI, E. J. - Seis anos de experiência com valva de dura-máter. Arq. Bras. Cardiol., 30 (Supl. 2): 173, 1977. (Resumo).

12 PUIG, L. B.; VERGINELLI, G.; SOSA, E. A.; ROMA, L. S. L.; GARCIA, D. P.; CONCEIÇĀO, A. N.; ZERBINI, E. J.; PILEGGI, F. - Avaliaçăo hemodinâmica de valva de dura-máter mitral e tricúspide. Arq. Bras. Cardiol., 29: 297-301, 1976.

13 ROBLES, A.; VAUGHAN, M.; LAU, J. K. - Long-term assessment of aortic valve replacement with autologous pulmonary valve. Ann. Thorac. Surg., 39:238 . 242, 1985.

14 ROSS, D. - Applications of homografts in clinical surgery. J. Cardiac. Surg., 1 (Supl.): 175-183, 1987.

15 ROSS, D. - Homograft replacement of the aortic valve. Lancet, 2: 487, 1962.

16 ROSS, D.: Pulmonary valve autotransplantation (The Ross operation). J. Cardiac Surg., 3 (Supl.): 313 . $319,1988$.

17 ROSS, D. - Replacement of aortic and mitral valves with a pulmonary autograft. Lancet, 2: $945-958$, 1967.

18 ROSS, D.; MARTELLI, V.; WAIN, W. H. - Allograft and autograft valves used for aortic valve replacement. In: IONESCU, M. I. (ed.) Tissue heart valves. London, Butterworth, 1979. p.127-172.

19 SPAMPINATO, N.; STASSANO, P.; CAMMAROTA, A. - Bioprosthesis at twelve years. J. Cardiac Surg. 3 (Supl.): 383-390, 1988. 
FURTADO, H. B.; DURAN, C. A.; MEJIAS, G. H.; BITTENCOURT, N.; BARROS, R. A.; CAMARGO, A. C. B.; GERMANO, A. E. - Transposiçāo da valva pulmonar para substituiçảo da valva aórtica. Rev. Bras. Cir. Cardiovasc, 5(3): 160$167,1990$.

STELZER, P. \& ELKING, R. C. - Homograft valves and conduit: application in cardiac surgery. Current Problems in Surgery, 26 (6), 1989.

\section{DISCUSSĀO}

\section{DR. PABLO POMERANTZEFF}

São Paulo, SP

Gostaria, inicialmente, de agradecer à Comissāo Organizadora pelo convite e parabenizar os autores pela brilhante apresentaçāo. Após receber o texto deste trabalho, minha primeira providência foi rever a anatomia e histologia das valvas aórtica e pulmonar. Segundo Testut, a circunferência do anel pulmonar é, em média, de $72 \mathrm{~mm}$ no homem e $68 \mathrm{~mm}$ na mulher, e a circunferência do orifício aórtico é de $70 \mathrm{~mm}$ no homem e $65 \mathrm{~mm}$ na mulher: portanto, o orifício pulmonar normal é praticamente igual, ou um pouco maior que o orifício aórtico normal. Com relação à vascularizaçāo, sabemos que apenas a mitral e a tricúspide têm uma pequena quantidade de vasos, estes nảo existindo nas valvas aórtica e mitral. Solicitei à Dra. Vera Aiello, do nosso Serviço de Anatomia Patológica, que fizesse duas lâminas, uma de valva pulmonar e outra de valva aórtica de um mesmo cadáver com valvas normais. Confirmando os dados de literatura, as valvas aórtica e pulmonar sāo iguais, sendo que a camada fibrosa envolvida por duas camadas elásticas é um pouco mais densa na valva aórtica. Os nódulos de Arâncio na valva aórtica são um pouco mais desenvolvidos que os nódulos de Mogagni da valva pulmonar, provavelmente pela diferença de pressão, já que são áreas de maior atrito e que impedem o deslisamento das válvulas entre si. Após os detaIhes de anatomia, fui rever a literatura e acredito ser importante salientar que, na procura de um substituto valvar ideal, os Drs. Lower, Stofer e Shumway, de Palo Alto, California, publicaram, em novembro de 1961, no Journal of Thoracic and Cardiovascular Surgery, trabalho experimental em cães nos quais substituíram a valva mitral por valva pulmonar autógena. Em 1967, Donald Ross publicou, no Lancet, os seus resultados em 14 pacientes em que realizou transposição da valva pulmonar, sendo 12 em posição aórtica e dois em posição mitral, e descreve a técnica que utilizou. Em 1970, GonzalesLavin, Greens e Ross publicam, no Surgery, os seus resultados com os primeiros 84 pacientes e salientam a indicaçāo prioritária em pacientes de menos de 40 anos. Destacam também, nesse trabalho, alguns aspectos de técnica cirúrgica, ressaltando a proximidade do primeiro ramo septal quan- do da dissecçāo da valva pulmonar e destacam, ainda, que em corte longitudinal as valvas aórtica e pulmonar sāo muito semelhantes. Em 1979, no livro Tissue Heart Valves, editado pelo Dr. lonesco, Ross e colaboradores relatavam resultados semeIhantes com dez anos de evoluçāo tardia com enxertos pulmonares autólogos e enxertos homólogos criopreservados. Em 1985, Robles, Ross e colaboradores em seu trabalho publicado no Annals of Thoracic Surgery, analisando a evolução de 14 anos dos enxertos pulmonares autógenos e comparando as mesmas aos seus resultados com enxerto homólogo e prótese de Starr-Edwards, demonstram, claramente, a superioridade dos enxertos autógenos. Para melhor poder avaliar a técnica operatória e para ter uma idéia sobre a dificuldade de tal procedimento, resolvi realizar a operaçāo em coraçāo de cadáver, sendo mais simples do que eu imaginava. Com relação ao tempo de CEC, sem dúvida deverá diminuir com o aumento da experiência, e acredito que, como defende o Dr. Henrique Furtado, a complexidade cirúrgica também nāo é problema difícil de se resolver. O ponto fundamental, como é muito bem ressaltado no trabalho, é o fato de estarmos implantando uma valva viva com todas as células intactas, inclusive com endotélio e fibroplastos. Penso que os maiores problemas estariam relacionados à adaptação da valva pulmonar em aorta de grande calibre e que os resultados tardios poderiam depender do tubo de pericárdio colocado em via de saída do ventrículo direito. Evidentemente, gostaríamos todos de analisar resultados de um maior número de casos, o que, tenho certeza, acontecerá nos próximos anos. Mais uma vez, parabéns aos autores e obrigado.

\section{DR. FURTADO \\ (Encerrando)}

Desejo agradecer à Comissão Organizadora, pela honra de ter apresentado este trabalho. Agradeço ao Dr. Pablo, pela sua dedicação ao fazer sua análise, com colocaçōes brilhantes e acrescentando importantes dados. Com relaçāo ao uso do tubo valvulado de pericárdio bovino na VSVD, acredito que, em virtude do baixo regime pressórico, nāo teremos problemas importantes e, caso haja, poderemos operá-lo, com baixa morbi-mortalidade. Também estamos estudando outras alternativas para se empregar na VSVD e, breve, colocaremos em uso clínico. Tenho consciência de que, à realizaçāo pioneira desta cirurgia na América Latina, seguirse-ão experiências de outros grupos que aumentarão a experiência neste campo. Muito Obrigado. 